\title{
Broadband and Highly Directional Visible Light Scattering by Laser-Splashed Lossless $\mathrm{TiO}_{2}$ Nanoparticles
}

\author{
Yinan Zhang ${ }^{1,2, *}$, Shiren Chen ${ }^{3}$ and Jing Han ${ }^{3}$ \\ 1 Institute of Photonic Chips, University of Shanghai for Science and Technology, Shanghai 200093, China \\ 2 Centre for Artificial-Intelligence Nanophotonics, School of Optical-Electrical and Computer Engineering, \\ University of Shanghai for Science and Technology, Shanghai 200093, China \\ 3 Institute of Photonics Technology, Jinan University, Guangzhou 510632, China; \\ chen.shiren@foxmail.com (S.C.); hanjing@stu2018.jnu.edu.cn (J.H.) \\ * Correspondence: zhangyinan@usst.edu.cn
}

Citation: Zhang, Y.; Chen, S.; Han, J. Broadband and Highly Directional Visible Light Scattering by LaserSplashed Lossless $\mathrm{TiO}_{2}$ Nanoparticles. Molecules 2021, 26, 6106. https:// doi.org/10.3390/molecules26206106

Academic Editors: Kai Chen, Arif Engin Çetin, Thu Hac Huong Le and Vladimir V. Kitaev

Received: 13 September 2021

Accepted: 8 October 2021

Published: 10 October 2021

Publisher's Note: MDPI stays neutral with regard to jurisdictional claims in published maps and institutional affiliations.

Copyright: ( $\odot 2021$ by the authors Licensee MDPI, Basel, Switzerland. This article is an open access article distributed under the terms and conditions of the Creative Commons Attribution (CC BY) license (https:// creativecommons.org/licenses/by/ $4.0 /)$.

\begin{abstract}
All-dielectric nanoparticles, as the counterpart of metallic nanostructures have recently attracted significant interest in manipulating light-matter interaction at a nanoscale. Directional scattering, as an important property of nanoparticles, has been investigated in traditional high refractive index materials, such as silicon, germanium and gallium arsenide in a narrow band range. Here in this paper, we demonstrate that a broadband forward scattering across the entire visible range can be achieved by the low loss $\mathrm{TiO}_{2}$ nanoparticles with moderate refractive index. This mainly stems from the optical interferences between the broadband electric dipole and the magnetic dipole modes. The forward/backward scattering ratio reaches maximum value at the wavelengths satisfying the first Kerker's condition. Experimentally, the femtosecond pulsed laser was employed to splash different-sized nanoparticles from a thin $\mathrm{TiO}_{2}$ film deposited on the glass substrate. Single particle scattering measurement in both the forward and backward direction was performed by a homemade confocal microscopic system, demonstrating the broadband forward scattering feature. Our research holds great promise for many applications such as light harvesting, photodetection and on-chip photonic devices and so on.
\end{abstract}

Keywords: directional scattering; dielectric nanoparticles; laser fabrication

\section{Introduction}

As the building block of metamaterials and metasurfaces, nanoparticles play a crucial role in controlling light propagation and regulating the light field distribution [1,2]. Although metal nanoparticles supporting localized surface plasmons have been widely investigated and are found in many applications such as Raman scattering enhancement [3], biosensing [4], metasurfaces [5], nonlinear plasmonics [6,7] and photovoltaics [8-10], their large optical losses and lack of magnetic resonances have hindered their further development. All-dielectric nanomaterials have recently emerged as a promising alternative to plasmonic nanoparticles in constituting high-efficiency photonic devices due to the coexistence of electric and magnetic resonances and lower optical losses as well.

Directional scattering is an important property in nanoparticles that can be used to make Huygens directional sources [11] and enhance solar energy harvesting [12,13] and photodetector responses. Previous studies have demonstrated that directional scattering is the result of amplitude and phase superposition due to interferences between different modes in the nanoparticles [14,15]. The research is mainly focused on high refractive index materials such as silicon [16], gallium nitride [17] and germanium [18]. Among them, silicon is the most widely used material due to its earth abundance and mature processing technologies. Andrey et al. reported the spattering of silicon particles by femtosecond laser ablation and observed the strong magnetic response and high-order dipole resonance [15]. Following this, Yuan Hsing Fu et al. observed the directional scattering behavior of silicon 
nanoparticles in the visible light range [19]. Later on, a series of work has been carried out to improve the directional scattering efficiency and its operation wavelengths by other mechanisms, such as the Fano resonance $[18,20]$ and the multibody enhanced electric field [21].

However, the high refractive index nanoparticles such as Si always have their electric dipole and magnetic dipole resonances separated from each other in the broad spectrum, and this leads to rather narrow band directional scattering. Although the non-negligible imaginary part of the high refractive index nanoparticles, such as Ge, can red- and blue-shift the electric and magnetic dipole resonances and make them closer, the large optical loss inevitably limits the directivity efficiency. With the refractive index reduced, the magnetic dipole resonance is shifted closer to the electric dipole modes and can potentially enhance the optical mode interaction and the corresponding directionality. It has been experimentally demonstrated that forwardly directional scattering behavior exists in copper oxide [22]. However, the relatively large optical loss derived from the non-negligible imaginary part of the refractive index of copper oxide and the large scattering strength difference between electric and magnetic dipole resonance empower their forward/backward scattering ratio at a low level.

Here, we proposed $\mathrm{TiO}_{2}$ nanoparticles for directing light scattering with a moderate real refractive index of $\sim 2.4$ and an imaginary part of zero in the visible range $[23,24]$. Through finite difference time domain (FDTD) calculation and Mie scattering theory $[25,26]$ analysis, we find that in the entire visible band, the forward scattering dominates with the forward/backward (F/B) scattering, with large tolerance on the particle morphology, reaching maximum value at the wavelength and satisfying the first Kerker's condition. Experimentally, we prepared $\mathrm{TiO}_{2}$ nanoparticles by a simple femtosecond laser ablation method $[16,27,28]$. Nanoparticles with diameters between $200 \mathrm{~nm}$ and $260 \mathrm{~nm}$ were successfully obtained on ITO substrates. Forward and backward scattering spectra were measured by a homemade dark-field scattering microscope attached to a spectrometer. The experimental results are in good agreement with the simulations, with the forward scattering dominating across the entire visible band.

\section{Results and Discussions}

Since the refractive index of the nanoparticles has noticeable effect on the light scattering behavior, we first performed a refractive index search for the directionality features of the nanoparticles, with the results shown in Figure 1a, where the F/B scattering ratio of a spherical nanoparticle with $200 \mathrm{~nm}$ diameter located in the air is shown. In this simulation, the imaginary part of the refractive index was set as zero to solely investigate the effect of the magnitude of the real part. A total-field scattered field light source was employed, and the boundary conditions were set as perfectly matched layers. The mesh size of the simulation region was $2 \mathrm{~nm}$, ensuring high accuracy simulation results. We can clearly observe a unique refractive index region $(2<\mathrm{n}<2.7)$, where the forward scattering dominates in the entire visible range, with distinct peaks for the F/B scattering ratio. When the refractive index is larger than 2.7, significant backward scattering starts to occur and light directionality in both forward and backward scattering occurs in the visible band. Indeed, previous reports have shown that high refractive index materials such as $\mathrm{Si}$ and Ge can introduce stronger scattering at both the forward and backward direction, dependent on the wavelengths. However, so far, very few research studies on the nanoparticle with moderate refractive index have been reported. Figure $1 b, c$ compare the scattering directionalities of the nanoparticles with a refractive index of 3.5 and 2.4, representing high and moderate refractive index materials. Clearly, for the high refractive index 3.5 , in the short wavelength range below $600 \mathrm{~nm}$ and long wavelength range above $700 \mathrm{~nm}$, the forward scattering outperforms backward scattering while in the range between 600 and $700 \mathrm{~nm}$, the backward scattering dominates. On the other hand, for the nanoparticles with a refractive index of 2.4, forward scattering is significantly larger than backward scattering in the entire visible range, with a maximum F/B ratio $>30$ at around $550 \mathrm{~nm}$. Since the 
directionalities stem from the multipolar interferences in the nanoparticles, we performed multipolar decomposition for the corresponding nanoparticle, with the results shown in Figure $1 \mathrm{~d}$,e, respectively. For high refractive index nanoparticles, ED and MD are separated from each other, and high-order modes EQ and MQ appear in the short band. The first and second Kerker's conditions are satisfied at around $800 \mathrm{~nm}$ and $680 \mathrm{~nm}$, leading to the dominating forward and backward scattering, respectively, at these two wavelengths. As the refractive index decreases to 2.4, ED and MD begin to overlap with each other and constructive interference occurs across the entire band, leading to an enhanced forward scattering. Figure $1 \mathrm{~d}$ shows the far-field scattering patterns of a $200 \mathrm{~nm} \mathrm{TiO}_{2}$ particle at three different wavelengths, corresponding to the positions of forward $(500 \mathrm{~nm})$ and backward $(480 \mathrm{~nm})$ scattering peaks and F/B peak $(550 \mathrm{~nm})$. It can be found that at these four different wavelengths, the $\mathrm{TiO}_{2}$ particles have significant forward directivity. Unlike high refractive index materials $\mathrm{Si}$ or $\mathrm{Ge}, \mathrm{TiO}_{2}$ maintains good directionality beyond the $\mathrm{F} / \mathrm{B}$ peak. This demonstrates that the moderate refractive index materials not only possess lower loss, but also show better directionality. Compared to the use of Fano resonance or multipolymer structure to enhance and regulate the directional behavior, moderate refractive index media only need to consider their own size and operating band.
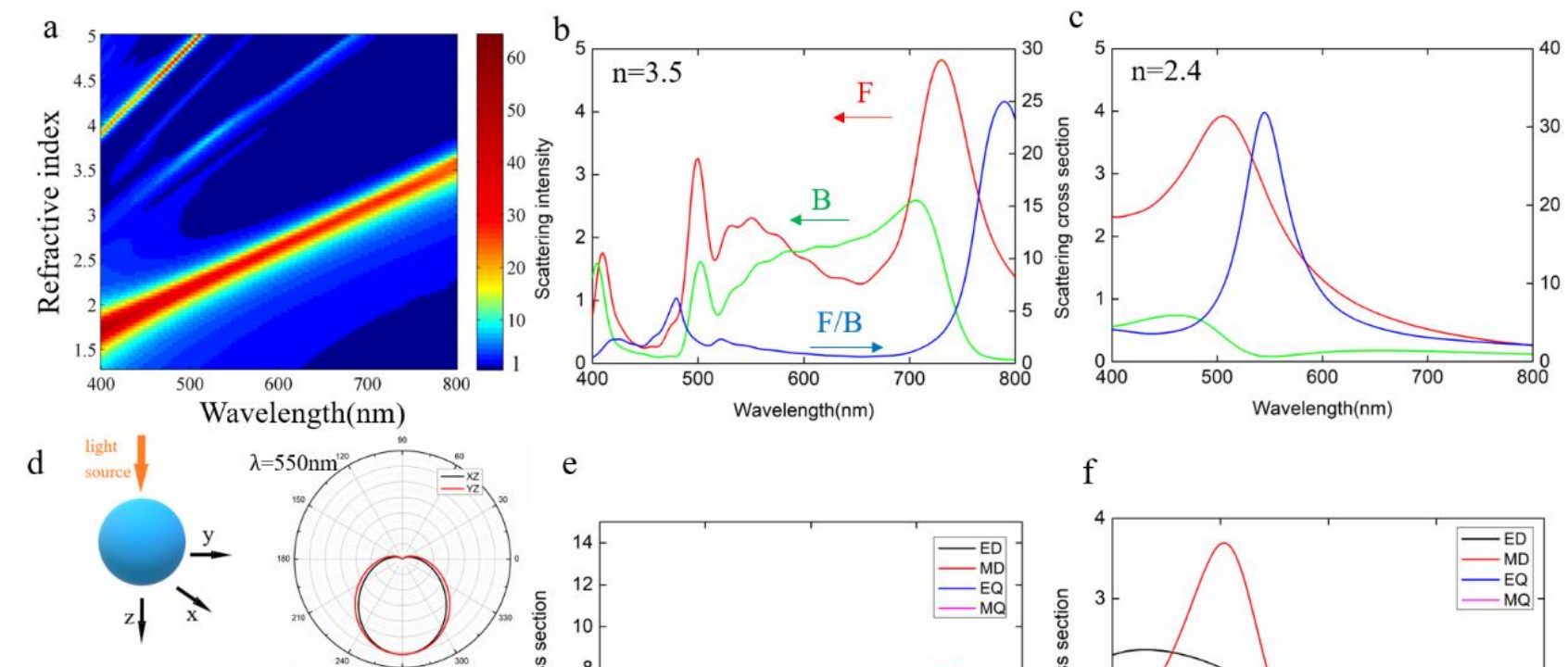

e
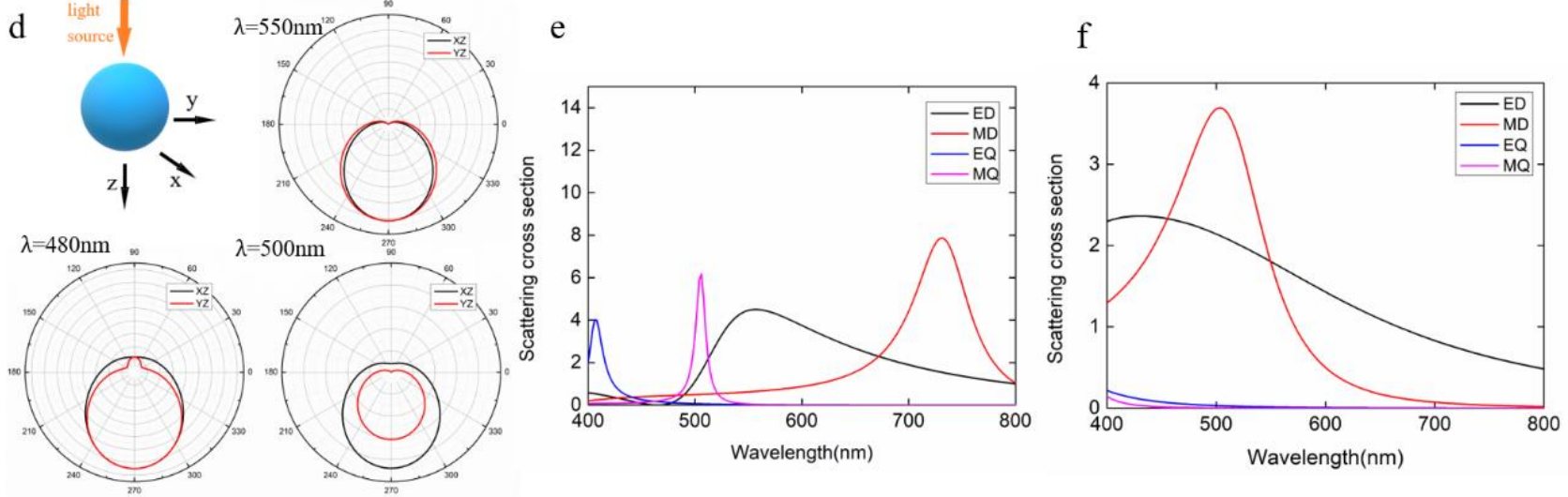

Figure 1. Effect of the refractive index on the forward/backward scattering. (a) The F/B value as a function of wavelength for spherical nanoparticles with a refractive index from 1.3 to 5. (b,c) Forward and backward scattering and F/B spectra for spherical nanoparticles with a refractive index of 3.5 and 2.4. (d) Far-field scattering patterns of $\mathrm{TiO}_{2}$ nanoparticles at the wavelengths of $480 \mathrm{~nm}, 500 \mathrm{~nm}$ and $550 \mathrm{~nm}$. (e,f) Multipolar decomposition by Mie scattering theory.

The size of nanoparticles has a significant influence on the scattering properties. Therefore, we calculated the $\mathrm{F} / \mathrm{B}$ value of spherical $\mathrm{TiO}_{2}$ nanoparticles with a radius from $80 \mathrm{~nm}$ to $150 \mathrm{~nm}$, with the results shown in Figure 2a. It can be seen that as the size increases, the F/B peak value gradually red-shifts over the entire visible spectral range. The F/ B value is always larger than one, demonstrating their broadband forward scattering feature. Considering the experimental fabricated nanoparticles are not always ideally spherically shaped, we simulated ellipsoidal-shaped nanoparticles with varied height $R_{z}$ $\left(40-150 \mathrm{~nm}\right.$ ) for a fixed $R_{x}$ and $R_{y}$ value of $100 \mathrm{~nm}$. As the value of $R_{z}$ increases, the 
F/B peak gradually red-shifts but the value is still larger than 1 through the entire visible spectral range. Therefore, $\mathrm{TiO}_{2}$ is a kind of forwardly directional scattering nanomaterial with large shape tolerance, favorable for stringent nanofabrication processes.
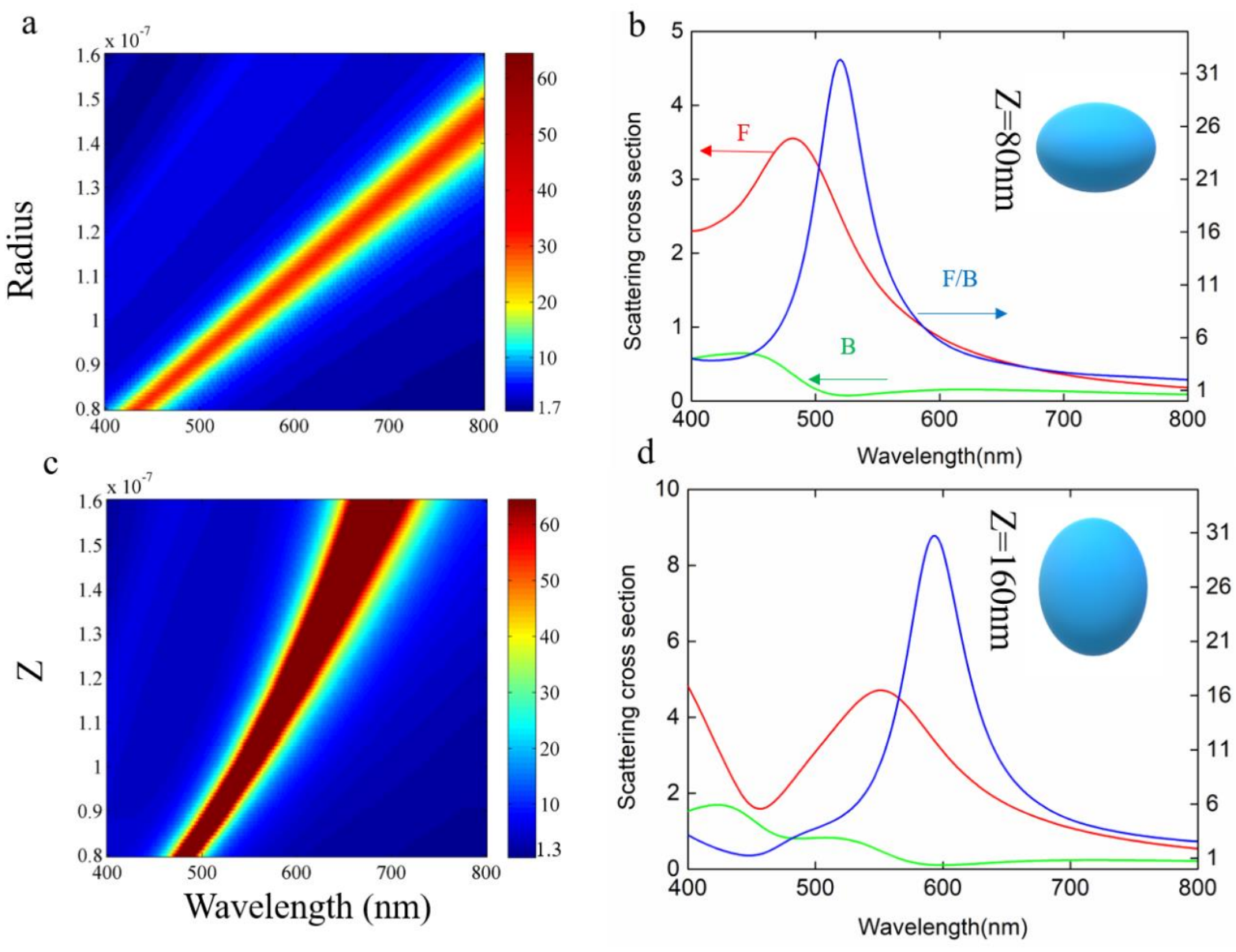

Figure 2. Shape effect of $\mathrm{TiO}_{2}$ nanoparticles on scattering. (a) The effect of the radius on the $\mathrm{F} / \mathrm{B}$ value with the color representing the F/B value. (b) The effect of the $\mathrm{Z}$ on the F/B value, with the color representing the F/B value. (c,d) Forward scatter and backscatter with the same radius, different $Z$ sizes and $F / B$ values.

In order to verify the scattering directionality of $\mathrm{TiO}_{2}$ nanoparticles, we used the fs pulsed laser ablation method to prepare $\mathrm{TiO}_{2}$ nanoparticles, similar to that used in [12]. Figure $3 \mathrm{a}$ is the schematic diagram of the process by which $\mathrm{TiO}_{2}$ nanoparticles were obtained. Thin film with a thickness of about $200 \mathrm{~nm}$ was deposited on a silicon wafer. Then an fs laser (Coherent, Libra) with a central wavelength of $800 \mathrm{~nm}$ and a frequency of $1000 \mathrm{HZ}$ was tightly focused on the $\mathrm{TiO}_{2}$ thin film surface by an objective lens with an NA $=0.7$. A point-by-point method was used to scan the sample. The exposure time was set as $100 \mathrm{~ms}$ and the average laser power was $0.5 \mathrm{~mW}$. Due to the local temperature instant rise by the high-intensity energy of fs pulses, a large quantity of nanoparticles was splashed and the ITO glass on top of the Si wafer with a certain distance was used to collect the spattered nanoparticles. ITO glass instead of normal glass was used for the purpose of imaging the nanoparticles using a conductive substrate in the scanning electron microscopy measurement.

Figure $3 b$ shows the SEM image of a selected area of the ITO glass distributed with varied sizes of nanoparticles. The lower left corner is the TEM image of an individual particle. The corresponding dark-field images in both forward and backward directions are shown in Figure 3c,d, respectively. Figure 3e is the Raman spectrum of the $\mathrm{TiO}_{2}$ nanoparticles that we made, demonstrating that the fs laser does not change the inherent 
properties of the $\mathrm{TiO}_{2}$ thin film. It can be seen from Figure $3 \mathrm{c}$ that the different particle colors cover the entire visible light region from blue to red as the size increases. From Figure $3 \mathrm{c}$ we can see that, unlike backward scattering, the color of the particles in the forward scattering dark-field image is red-shifted as a whole, and many particles become white-like colors. For this, we selected six different-sized particles (Figure 3f) with a diameter that gradually increased from $203 \mathrm{~nm}$ to $263 \mathrm{~nm}$. We used a self-made microscope system and spectrometer to collect the forward and back spectra of these nanoparticles and compared their forward/backward scattering.
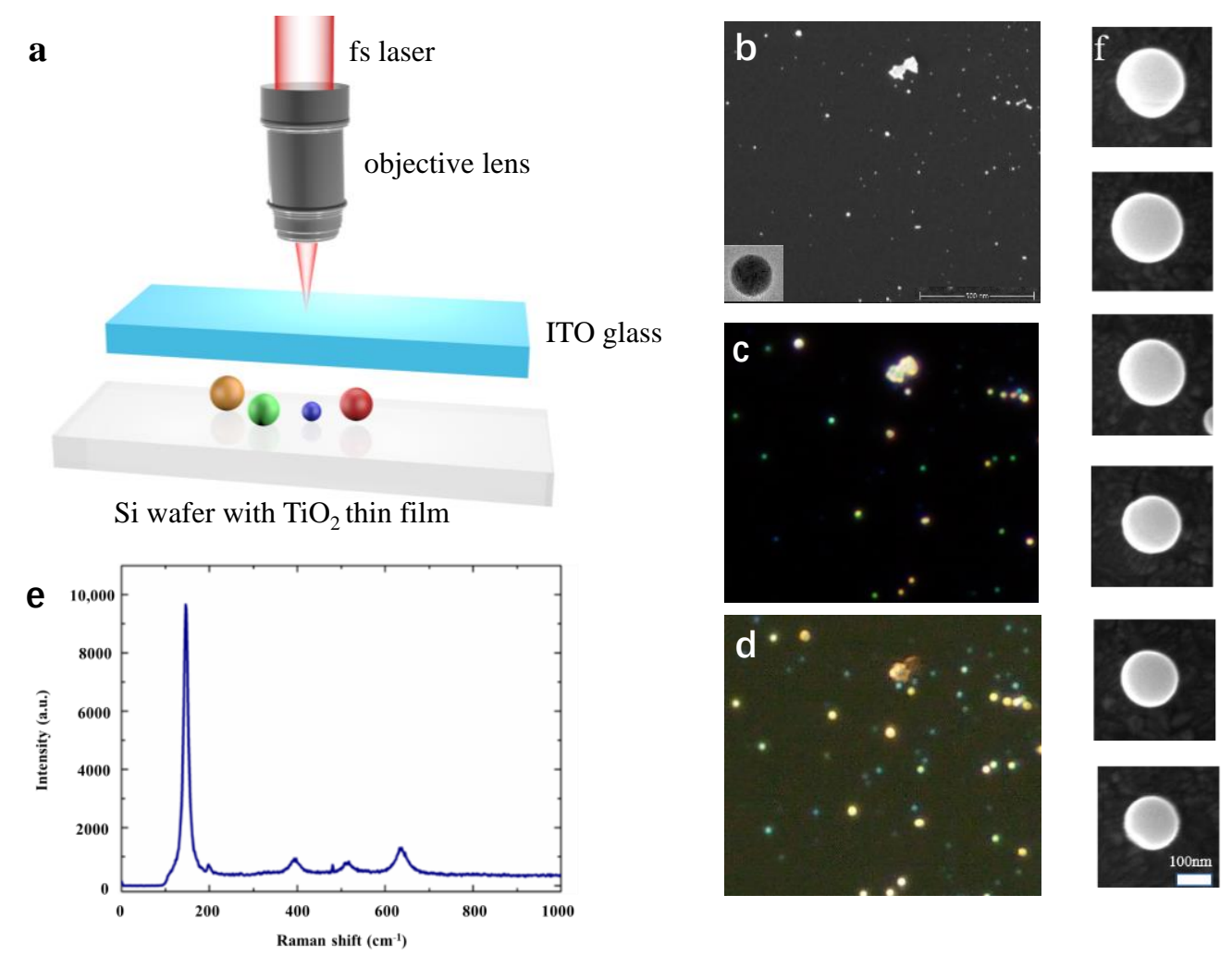

$263 \mathrm{~nm}$

$203 \mathrm{~nm}$

Figure 3. (a) Schematic of the laser-splashed process for nanoparticle fabrication. (b) SEM image of fabricated nanoparticles with the inset showing the TEM image of one representative nanoparticle. (c,d) Reflective and transmissive dark-field microscopy images of the areas corresponding to the SEM image area. (e) The Raman spectrum of one representative $\mathrm{TiO}_{2}$ nanoparticle. (f) SEM images of selected nanoparticles with various diameters from $203 \mathrm{~nm}$ to $263 \mathrm{~nm}$.

In order to obtain the forward/backward scattering ratio of the particles we obtained, we further performed spectral measurements on the selected six particles. Figure 4 presents the forward and backward scattering from the selected six individual nanoparticles and the forward/backward scattering ratio. The forward/backward scattering was normalized to the scattering by a subwavelength dust spot. The inset images in the figures are their reflection dark-field microscope images and transmission dark-field images, respectively, corresponding to backward scattering and forward scattering. It can be seen from Figure 4 that as the size increases, the backward scattering peak gradually red-shifts from $\sim 500 \mathrm{~nm}$ to $\sim 650 \mathrm{~nm}$, with the accompanying color appearance of the particles changing from blue to purple. The forward scattering curve also red-shifts as the size increases, with the peak value longer than that of the backward scattering, agreeing well with the simulation results. Furthermore, the spectra in forward scattering mostly locate at the red region with a broadspectrum distribution, corresponding to the yellow-white colors in the dark-field images. 

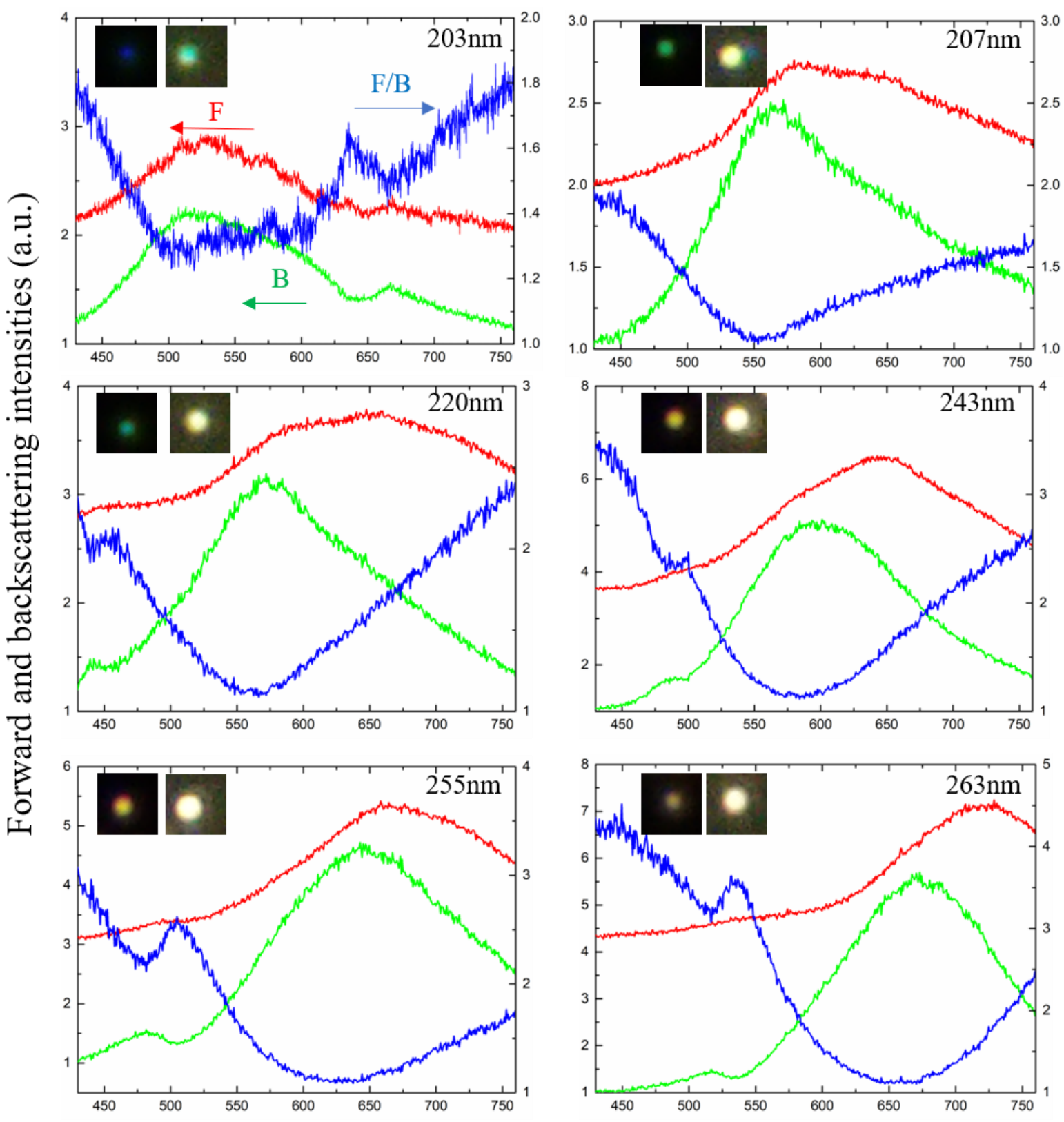

Wavelength $(\mathrm{nm})$

Figure 4. Experimentally measured forward and backward scattering spectra of six selected sizes of nanoparticles. Left axes show forward (red) and backward (green) scattering intensities, and right axes show F/B ratio (blue) of nanoparticles.

From the F/B values of different-sized nanoparticles measured in Figure 4, we can see $\mathrm{TiO}_{2}$ nanoparticles can achieve $\mathrm{F} / \mathrm{B}>1$ across the entire visible band between $400 \mathrm{~nm}$ and $800 \mathrm{~nm}$. The F/B peak gradually red-shifts as the size increases. It is noted that experimental F/B is relatively small, with a maximum value of $\sim 4.5$. This is probably because the transmitted light source passes through an attenuator and is incident on the ITO glass with a certain absorption, which leads to a decrease in relative intensity and a slight shift in peak value. Unlike the simulation, the experimentally measured F/B value also has an ascending process in the range of $650-750 \mathrm{~nm}$, which is related to the chromatic aberration that may be caused by the lens combination during absorption and measurement of the ITO material at different wavelengths. Nevertheless, the basic trend of broadband forward scattering domination in the visible range is still consistent with the predictions from simulations. 


\section{Conclusions}

In summary, we have numerically calculated the forward/backward scattering of $\mathrm{TiO}_{2}$ nanoparticles and their ratio in the entire visible region. Anisotropic scattering with the forward part dominating was found in the entire visible range with large tolerance on the particle shape and size. This is owing to the multipolar interference between the broadband $\mathrm{ED}$ and MD modes in the $\mathrm{TiO}_{2}$ nanoparticles. It is different from the polymer-enhanced electric field and the Fano resonance between the particles to enhance and regulate the $\mathrm{F} / \mathrm{B}$. Meanwhile, the imaginary part of $\mathrm{TiO}_{2}$ nanoparticles in the visible light band is zero, so the optical loss is extremely low. Experimentally, we prepared different sizes of $\mathrm{TiO}_{2}$ nanoparticles by the laser ablation method and measured the forward and backward scattering spectra using a homemade microscope system to verify our calculations. Our work has contributed to the determination of directional scattering conditions in the full visible band, and confirmed the broadband directional scattering properties of lossless $\mathrm{TiO}_{2}$ nanoparticles.

Author Contributions: Conceptualization, Y.Z.; methodology, Y.Z., S.C.; software, S.C.; validation, S.C.; formal analysis, Y.Z., S.C.; investigation, Y.Z., S.C.; resources, Y.Z.; data curation, S.C.; writingoriginal draft preparation, S.C., Y.Z.; writing-review and editing, Y.Z., J.H.; visualization, S.C.; supervision, Y.Z.; project administration, Y.Z.; funding acquisition, Y.Z. All authors have read and agreed to the published version of the manuscript.

Funding: This research was funded by National Natural Science Foundation of China (NSFC) (Grant No. 62175154), Shanghai Pujiang Program (20PJ1411900), Shanghai Science and Technology Program (21ZR1445500), Guangdong Basic and Applied Basic Research Foundation (2020A1515011510).

Institutional Review Board Statement: Not applicable.

Informed Consent Statement: Not applicable.

Data Availability Statement: Not applicable.

Acknowledgments: Yinan Zhang acknowledges the support by the Program for Professor of Special Appointment (Eastern Scholar) at Shanghai Institutions of Higher Learning. This work is also supported by National Natural Science Foundation of China (NSFC) (Grant No. 62175154), Shanghai Pujiang Program (20PJ1411900), Shanghai Science and Technology Program (21ZR1445500), Guangdong Basic and Applied Basic Research Foundation (2020A1515011510).

Conflicts of Interest: The authors declare no conflict of interest.

Sample Availability: Not applicable.

\section{References}

1. Hillenbrand, R.; Taubner, T.; Keilmann, F. Phonon-enhanced light-matter interaction at the nanometre scale. Nature 2002, 418, 159-162. [CrossRef]

2. Wang, S.; Li, X.; Deng, Z.-L.; Cao, Y.; Hu, D.; Xu, Y.; Cai, B.; Jin, L.; Bao, Y.; Wang, X. Angular Momentum-Dependent Transmission of Circularly Polarized Vortex Beams Through a Plasmonic Coaxial Nanoring. IEEE Photonics J. 2018, 10, 1-9. [CrossRef]

3. Qian, X.; Peng, X.-H.; Ansari, O.D.; Yin-Goen, Q.; Chen, G.Z.; Shin, D.M.; Yang, L.; Young, A.N.; Wang, M.D.; Nie, S. In vivo tumor targeting and spectroscopic detection with surface-enhanced Raman nanoparticle tags. Nat. Biotechnol. 2008, 26, 83-90. [CrossRef] [PubMed]

4. Liu, J.; Lu, Y. Preparation of aptamer-linked gold nanoparticle purple aggregates for colorimetric sensing of analytes. Nat. Protoc. 2006, 1, 246-252. [CrossRef]

5. Chen, L.M.; Liu, Y.N. Surface-enhanced Raman detection of melamine on silver-nanoparticle-decorated silver/carbon nanospheres: Effect of metal ions. ACS Appl. Mater. Interfaces 2011, 3, 3091-3096. [CrossRef] [PubMed]

6. Zhang, Y.; Han, J.; Shi, L.; Chen, S.; Feng, Z.; Lu, H.; Gu, M.; Li, X. Extremely Polarized and Efficient Hot Electron Intraband Luminescence from Aluminum Nanostructures for Nonlinear Optical Encoding. Laser Photonics Rev. 2021, 15, 2000339. [CrossRef]

7. Zhu, Z.; Shi, L.; Chen, S.; Han, J.; Zhang, H.; Li, M.; Hao, H.; Luo, J.; Wang, X.; Gu, B.; et al. Enhanced second harmonic emission with simultaneous polarization state tuning by aluminum metal-insulator-metal cross nanostructures. Opt. Express 2019, 27, 30909-30918. [CrossRef] [PubMed]

8. Zhang, Y.; Ouyang, Z.; Stokes, N.; Jia, B.; Shi, Z.; Gu, M. Low cost and high performance Al nanoparticles for broadband light trapping in Si wafer solar cells. Appl. Phys. Lett. 2012, 100, 151101. [CrossRef] 
9. Zhang, Y.; Jia, B.; Gu, M. Biomimetic and plasmonic hybrid light trapping for highly efficient ultrathin crystalline silicon solar cells. Opt. Express 2016, 24, A506. [CrossRef]

10. Zhang, Y.; Chen, X.; Cai, B.; Luan, H.; Zhang, Q.; Gu, M. Photonics Empowered Passive Radiative Cooling. Adv. Photonics Res. 2021, 2, 2000106. [CrossRef]

11. Liberal, I.; Ederra, I.; Gonzalo, R.; Ziolkowski, R. Induction Theorem Analysis of Resonant Nanoparticles: Design of a Huygens Source Nanoparticle Laser. Phys. Rev. Appl. 2014, 1, 044002. [CrossRef]

12. Zhang, Y.; Xu, Y.; Chen, S.; Lü, H.; Chen, K.; Cao, Y.; Miroshnichenko, A.E.; Gu, M.; Li, X. Ultra-Broadband Directional Scattering by Colloidally Lithographed High-Index Mie Resonant Oligomers and Their Energy-Harvesting Applications. ACS Appl. Mater. Interfaces 2018, 10, 16776-16782. [CrossRef]

13. Zhang, Y.; Chen, S.; Hu, D.; Xu, Y.; Wang, S.; Qin, F.; Cao, Y.; Guan, B.; Miroshnichenko, A.; Gu, M.; et al. Coloring solar cells with simultaneously high efficiency by low-index dielectric nanoparticles. Nano Energy 2019, 62, 682-690. [CrossRef]

14. Gómez-Medina, R.; Garcia-Camara, B.; Suárez-Lacalle, I.; González, F.; Moreno, F.; Nieto-Vesperinas, M.; Sáenz, J.J. Electric and magnetic dipolar response of germanium nanospheres interference effects, scattering anisotropy, and optical forces. $J$. Nanophotonics 2011, 5, 30-32. [CrossRef]

15. Evlyukhin, A.B.; Novikov, S.M.; Zywietz, U.; Eriksen, R.L.; Reinhardt, C.; Bozhevolnyi, S.I.; Chichkov, B.N. Demonstration of magnetic dipole resonances of dielectric nanospheres in the visible region. Nano Lett. 2012, 12, 3749-3755. [CrossRef] [PubMed]

16. Zywietz, U.; Evlyukhin, A.; Reinhardt, C.; Chichkov, B. Laser printing of silicon nanoparticles with resonant optical electric and magnetic responses. Nat. Commun. 2014, 5, 3402. [CrossRef]

17. Person, S.; Jain, M.; Lapin, Z.; Sáenz, J.J.; Wicks, G.; Novotny, L. Demonstration of zero optical backscattering from single nanoparticles. Nano Lett. 2013, 13, 1806-1809. [CrossRef] [PubMed]

18. Ma, C.; Yan, J.; Huang, Y.; Yang, G. Directional Scattering in a Germanium Nanosphere in the Visible Light Region. Adv. Opt. Mater. 2017, 5, 1700761. [CrossRef]

19. Fu, Y.H.; Kuznetsov, A.; Miroshnichenko, A.; Yu, Y.F.; Luk'Yanchuk, B. Directional visible light scattering by silicon nanoparticles. Nat. Commun. 2013, 4, 1527. [CrossRef]

20. Wiecha, P.R.; Cuche, A.; Kallel, H.; Francs, G.C.D.; Lecestre, A.; Larrieu, G.; Larrey, V.; Fournel, F.; Baron, T.; Arbouet, A.; et al. Fano-Resonances in High Index Dielectric Nanowires for Directional Scattering. Phys. Appl. 2018, 283-309. [CrossRef]

21. Yan, J.H.; Liu, P.; Lin, Z.; Wang, H.; Chen, H.J.; Wang, C.X.; Yang, G.W. Magnetically induced forward scattering at visible wavelengths in silicon nanosphere oligomers. Nat. Commun. 2015, 6, 7042. [CrossRef] [PubMed]

22. Zhang, S.; Jiang, R.; Xie, Y.M.; Ruan, Q.; Yang, B.; Wang, J.; Lin, H.Q. Colloidal Moderate-Refractive-Index Cu $2 \mathrm{O}$ Nanospheres as Visible-Region Nanoantennas with Electromagnetic Resonance and Directional Light-Scattering Properties. Adv. Mater. 2015, 27, 7432-7439. [CrossRef]

23. Jankuj, J. The normal inhomogeneity studies of the refractive index in titanium dioxide films. Czechoslov. J. Phys. B 1986, 36, 855-862. [CrossRef]

24. Zhao, Z.; Tay, B.K.; Yu, G. Room-temperature deposition of amorphous titanium dioxide thin film with high refractive index by a filtered cathodic vacuum arc technique. Appl. Opt. 2004, 6, 1281-1285. [CrossRef] [PubMed]

25. Van de Hulst, H.C. Light Scattering by Small Particles. Q. J. R. Meteorol. Soc. 1957, 84, 198-199. [CrossRef]

26. Zhao, Q.; Zhou, J.; Zhang, F.; Lippens, D. Mie resonance-based dielectric metamaterials. Mater. Today 2009, 12, 60-69. [CrossRef]

27. Zhang, Y.; Shi, L.; Hu, D.; Chen, S.; Xie, S.; Lu, Y.; Cao, Y.; Zhu, Z.; Jin, L.; Guan, B.-O.; et al. Full-visible multifunctional aluminium metasurfaces by in situ anisotropic thermoplasmonic laser printing. Nanoscale Horiz. 2019, 4, 601-609. [CrossRef]

28. Hu, D.; Lu, Y.; Cao, Y.; Zhang, Y.; Xu, Y.; Li, W.; Gao, F.; Cai, B.; Guan, B.-O.; Qiu, C.-H.; et al. Laser Splashed Three-Dimensional Plasmonic Nanovolcanoes for Steganography in Angular Anisotropy. ACS Nano 2018, 12, 9233-9239. [CrossRef] [PubMed] 